\title{
Metallic Nano-Rings for Efficient, Broadband Light Extraction from Solid-State Single-Photon Sources
}

\author{
Oliver J. Trojak ${ }^{1}$, C. S. Woodhead ${ }^{2}$, J. D. Song ${ }^{3}$, R. J. Young ${ }^{2}$, L. Sapienza ${ }^{1}$ \\ ${ }^{I}$ Department of Physics and Astronomy, University of Southampton, SO17 1BJ, Southampton, United Kingdom \\ ${ }^{2}$ Physics Department, Lancaster University, Lancaster LA1 4YB, UK \\ ${ }^{3}$ Centre for Opto-Electronic Materials and Devices Research, KIST, Seoul 136-791, South Korea \\ Author e-mail address: o.trojak@soton.ac.uk, l.sapienza@soton.ac.uk
}

\begin{abstract}
Metallic nano-rings deposited on the sample surface, centered around single InAs/GaAs quantum dots, enhance the brightness of the emission up to $\times 20$, further enhanced by $\times 10$ by epoxy solid-immersion lenses, in a scalable broadband device.

OCIS codes: (170.6280) Spectroscopy, fluorescence and luminescence; (220.0220) Nanostructure fabrication; (240.3990)

Micro-optical devices; (250.5590) Quantum-well, -wire and -dot devices; (300.6470) Spectroscopy, semiconductors
\end{abstract}

\section{Introduction}

Bright and pure single-photon sources are an essential component of emerging quantum technologies and solidstate single-photon emitters are the most promising candidates for integration in on-chip devices $[1,2]$. However, such emitters can suffer from poor light extraction efficiencies, because of the large difference in refractive index between the bulk semiconductor and air, that results in only a small fraction (down to less than 1\%) of the emitted photons reaching free-space collection optics, due to total internal reflection. In order to overcome this issue, photonic devices, such as micro-pillars, nanowires and suspended gratings have been developed [1]. Such devices show extraction efficiencies reaching up to about $70 \%$ but present narrow operation bandwidths and often require relative tuning of the emitter/cavity emission wavelengths to achieve resonance conditions.

Here, we present a broadband, scalable device based on a metallic nano-ring deposited on the sample surface, centered around a single InAs/GaAs quantum dot (QD). This device allows the brightness of single emission lines to be increased up to a factor of 20 [3]. We also show how such a device can be combined with deterministically positioned solid immersion lenses (SILs) that provide a further, cumulative enhancement of up to a factor of 10 in the brightness of the emitted light [4].

\section{Discussion of the results}

The dimension of metallic nano-rings, centered in correspondence to an emitting dipole (Fig. 1a), are optimized using finite-difference time-domain (FDTD) simulation techniques. As shown in Fig. 1c-h, the metallic nano-ring acts as a lens that focuses the emitted light towards the collection optics, thus increasing the single-photon extraction efficiency. Gold rings, of dimensions of $220 \mathrm{~nm}$ inner radius, $540 \mathrm{~nm}$ outer radius and $67 \mathrm{~nm}$ thickness, are then fabricated, by means of aligned e-beam lithography, thermal evaporation and chemical lift-off, on a sample consisting of a GaAs wafer embedding a single layer of low density $\left(\sim 1000 \mu \mathrm{m}^{-2}\right)$ InAs QDs, grown by molecular beam epitaxy and capped by a $95 \mathrm{~nm}$ GaAs layer. A scanning electron micrograph of a fabricated device in shown in Fig. $1 b$.

A double light-emitting diode (LED) imaging technique is used to locate single QDs with nanometer-scale accuracy [5]. The emitters are then spectrally characterized by means of micro-photoluminescence laser spectroscopy at cryogenic temperatures. The emission intensity is measured as a function of laser excitation power before and after the metallic ring is placed around a single InAs QD. As shown in Fig. 2a, the intensity of a single QD emission line is enhanced by the nano-ring by a factor $\sim 20$.

To further increase the brightness, a super-SIL formed by dispensing liquid ultraviolet-curable epoxy (with refractive index of 1.54) is placed on the sample in correspondence to the nano-rings (see Fig. 2b) [4]. The dispensing environment is filled with a liquid phase medium (glycerol), designed to increase the contact angle between the droplet edge and the substrate by modification of the interfacial surface tensions and, subsequently, the lens is solidified by exposure to UV light. The effect of the super-SIL on the emission from a selected QD is shown in Fig 2c: the super-SIL further enhances the extraction of light, providing a photon flux on the first lens as high as $900 \mathrm{kHz}$, from an emitter in bulk.

\section{Conclusions}

We have shown how deterministically placed metallic nano-rings and epoxy super-SILs can be implemented to enhance the extraction efficiency from single QDs. We have measured enhancements in the brightness of single QD 
emission lines by the metallic nano-rings as high as $\times 20$ and by the super-SIL as high as $\times 10$ : potentially, for optimally placed nano-rings and SILs, the cumulative effect could therefore reach enhancement factors as high as a factor 200 .

We have implemented such a combined system to show bright emission from single QD lines, in a scalable and relatively easy to fabricate device: the positioning of single QDs can be carried out in an automated system, the dimensions of the rings are compatible with photolithography and nano-imprint, and the super-SIL deposition requires only optical microscopy tools.

The combined enhancement effect of the nano-ring and the super-SIL is wavelength insensitive, therefore broadband, and compatible with any kind of solid-state emitter of classical or quantum light, on any substrate.
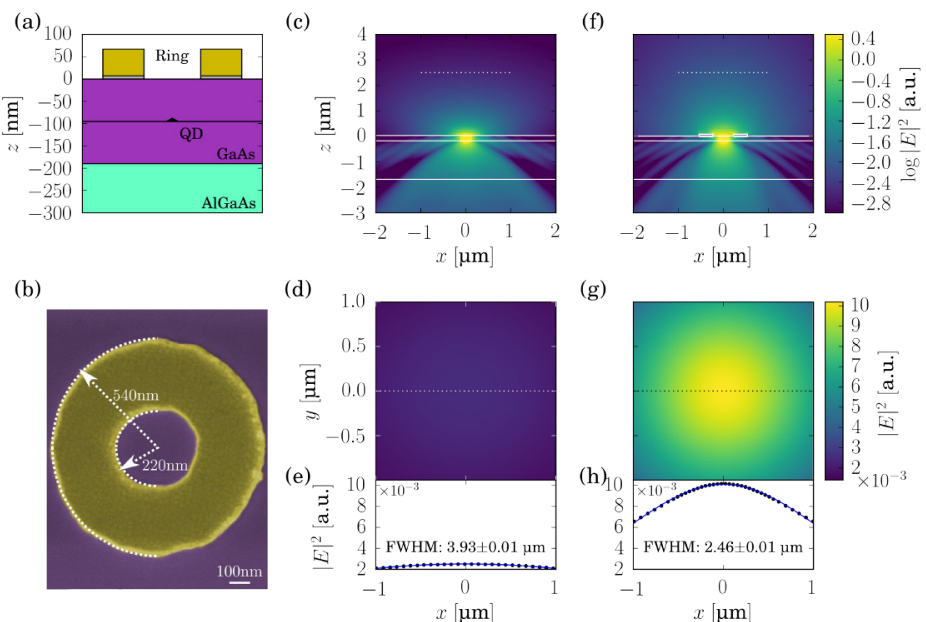

Figure 1: (a) Schematic of the sample (not to scale). (b) False colour scanning electron micrograph of a gold nano-ring, with dimensions shown. (c) FDTD simulation of the electric field from a dipole emitter placed at the QD position indicated in (a). The solid white lines indicate the layers structure. (d) Plot of the electric field profile measured $2.5 \mu \mathrm{m}$ from the sample surface, along the dotted line shown in (c). (e) Linecut of the farfield profile (symbols), taken across the dotted line shown in (d). The full-width half-maximum (FWHM) of the fit (blue line) is shown, too. (f-h) Same as (c-e), when a metallic nano-ring is present on the sample surface, centered around the emitter position.
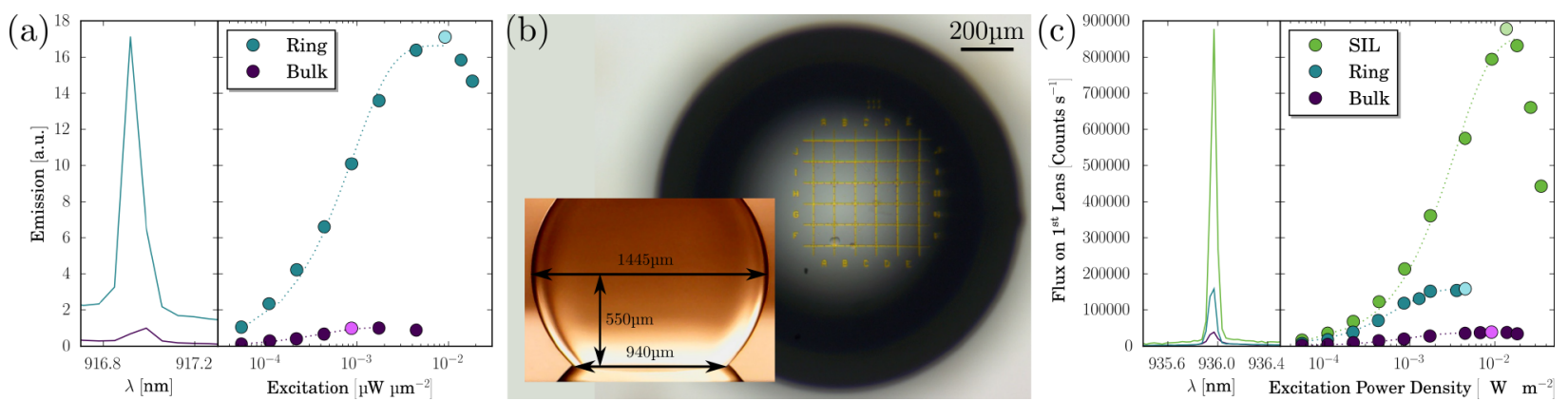

Figure 2: (a) Left: Photoluminescence spectra collected at saturation pump power for the emitter in bulk (violet line) and after ring deposition (blue line). Right: Intensity of the emission lines shown in left panel plotted as a function of laser excitation power density (lighter dots correspond to the peak intensity from the spectra shown in the left panel). (b) Optical micrograph of a super-SIL deposited on the InAs/GaAs QD sample under study, in correspondence to metallic alignment marks and nano-rings. Inset: side profile of an epoxy super-SIL with critical dimensions shown. (c) Same as (a), but showing emission from a QD in bulk, with a nano-ring placed on the sample surface, centered around the emitter's position and with nano-ring and super-SIL deposited on the sample's surface (violet, blue and green symbols and lines, respectively).

\section{References}

[1] I. Aharonovich, D. Englund, M. Toth, "Sold-state single-photon emitters", Nature Photonics 10, 631-641 (2016)

[2] O. Gazzano, G. Solomon, "Toward optical quantum information processing with quantum dots coupled to microstructures", Journal of the Optical Society of America B 33, C160-C175 (2016)

[3] O. J. Trojak, S. I. Park, J. D. Song, L. Sapienza, "Metallic nanorings for broadband, enhanced extraction of light from solid-state emitters", Applied Physics Letters B111B, 021109 (2017)

[4] C. S. Woodhead, J. Roberts, Y. J. Noori, Y. Cao, R. Bernado-Gavito, P. Tovee, A. Kozikov, K. Novoselov, R. J. Young, "Increasing the light extraction and longevity of TMDC monolayers using liquid formed micro-lenses", 2D Materials 4, 015032 (2016)

[5] L. Sapienza, M. Davanço, A. Badolato, K. Srinivasan, "Nanoscale optical positioning of single quantum dots for bright and pure singlephoton emission", Nature Communications 6, 7833 (2015) 